\title{
PIV Measurement of Turbulence Over a Streamwise Preferential Porous Medium
}

\author{
M. Morimoto ${ }^{1 *}$, Y. Okazaki ${ }^{1}$, Y. Kuwata ${ }^{1}$ and K. Suga ${ }^{1}$ \\ ${ }^{1}$ Osaka Prefecture University, Dep. of Mechanical Engineering, Sakai, Osaka, Japan \\ *morimoto@htlab.me.osakafu-u.ac.jp
}

\begin{abstract}
This study examines the possibility of orthotropic porous medium whose streamwise permeability is larger than the wall-normal permeability to reduce turbulent friction inspired by recent numerical studies of Rosti et al. (2018); Gómez-de Segura and García-Mayoral (2019). Because Gómez-de Segura and García-Mayoral (2019) used Brinkman equation to approximate the flow in the porous media, it is uncertain that such porous media really reduce the friction. We make a layered porous medium, which satisfies the drag reducing condition suggested by Gómez-de Segura and García-Mayoral (2019), and carry out particle image velocimetry measurements of turbulent square duct flows over it and examine the drag reduction probability. From the analyses of the obtained data, it is found that the friction on the porous-wall is nearly the same as that of the smooth-wall at $\operatorname{Re}_{b}<10000$ and tends to increase at $\operatorname{Re}_{b}>10000$.
\end{abstract}

\section{Introduction}

Understanding the turbulent flow physics over permeable porous media is of great importance from the engineering view point. Many researchers hence have dedicated to elucidate the characteristics of such flows (e.g. Lovera and Kennedy, 1969; Zippe and Graf, 1983; Breugem et al., 2006; Pokrajac and Manes, 2009, Manes et al., 2009; Suga et al., 2010, 2011, 2017; Manes et al., 2011; Suga, 2016). From those studies, it is well known that the wall permeability usually increases near-wall turbulence. Rosti et al. (2018); Gómez-de Segura and García-Mayoral (2019), however, reported numerical studies which indicated turbulent dragreduction over anisotropic porous media with a high streamwise permeability. Since the flows inside porous media of those numerical studies were not exactly treated but modelled by the Brinkman equation (Gómezde Segura and García-Mayoral, 2019) or were not solved by using idealized surface boundary conditions (Rosti et al., 2018), the realizability of their proposed drag reducing conditions has never been fully convinced yet.

Therefore, we have performed PIV experiments of turbulent square duct flows over a porous medium at the bulk Reynolds numbers of 3000-15000. The flow facility is the same as that used in the previous study (Suga et al., 2020) of our group. To satisfy the proposed drag reducing conditions, we have designed the structure of the porous medium. The porosity of the medium is 0.9 and the ratio of the square roots of the streamwise to wall-normal permeabilities is 4.3. This ratio well satisfies the drag reduction condition suggested by Rosti et al. (2018); Gómez-de Segura and García-Mayoral (2019). From the analyses of the obtained experimental data, which cover the drag reduction range of the wall-normal permeability Reynolds number suggested by Gómez-de Segura and García-Mayoral (2019), we have discussed whether the porous medium has the drag-reducing ability.

\section{Experimental method}

Figure 1(a) illustrates the experimental flow facility. The working fluid is tap water and the flow is conditioned in a conditioning tank and fully developed in a $3 \mathrm{~m}$ long drive section. Then the flow enters a $1 \mathrm{~m}$ long test section whose cross-sectional view is shown in figure 1(b). The test section, whose cross-section is $50 \mathrm{~mm}$ (height, $H$ ) $\times 50 \mathrm{~mm}$ (width), consists of solid smooth acrylic top and side walls and a porous bottom wall of $10 \mathrm{~mm}$ thickness. 


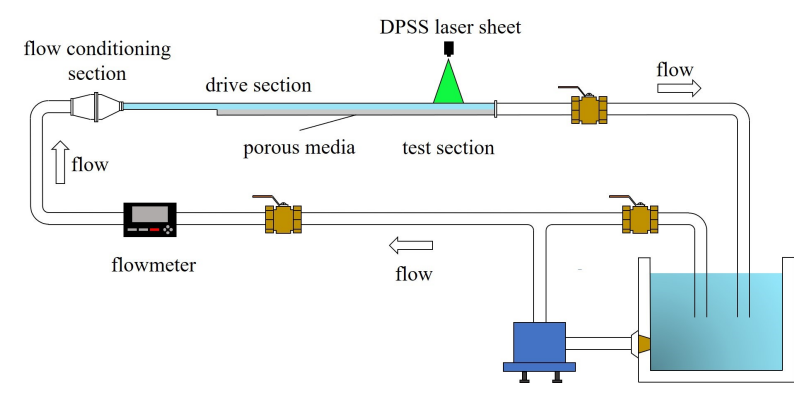

(a) Flow facility

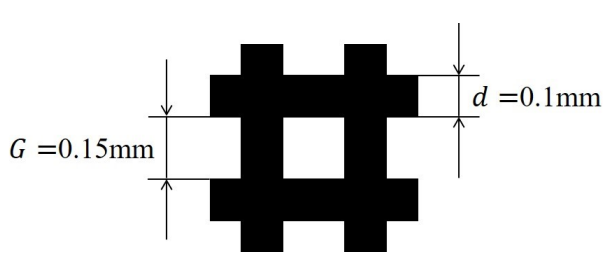

(c) Size of the mesh

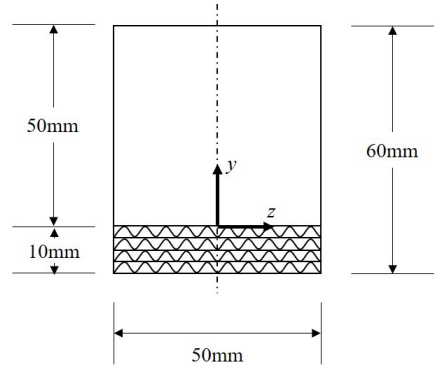

(b) Cross-sectional view

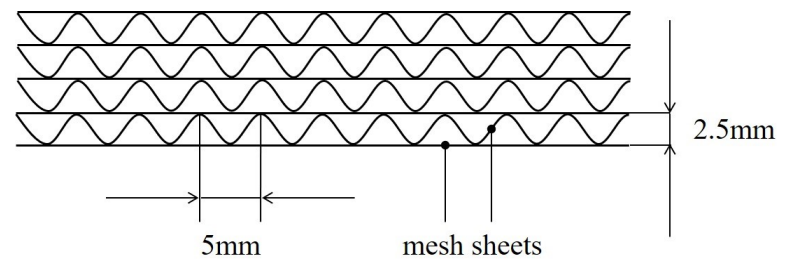

(d) Structure of the porous medium

Figure 1: Schematics of the experimental facility and the details of the porous medium.

The porous medium is made of stainless-steel woven-wire-mesh-sheets whose mesh spacing and wire diameter are $G=0.15 \mathrm{~mm}$ and $d=0.1 \mathrm{~mm}$, respectively, as illustrated in figure 1(c). The porous structure looks like a cardboard structure: corrugated mesh-sheets are sandwiched by flat mesh-sheets as illustrated in figure 1(d), The characteristics of the porous medium are listed in table 1 . The porosity $\varphi$ is obtained by measuring the weight and the mass of the porous medium. The diagonal component of the permeability tensor $K_{\alpha \alpha}$, is measured from the relation between the pressure drops and the flow rates through the porous medium stuffed into the horizontal duct flow facility. The permeability ratio parameter is defined as $\psi_{\alpha \beta}=$ $\sqrt{K_{\alpha \alpha} / K_{\beta \beta}}$.

Table 1: Characteristics of the porous medium.

\begin{tabular}{cccccc}
$\varphi$ & $\psi_{x y}$ & $\Psi_{z y}$ & $K_{x x}\left[\mathrm{~mm}^{2}\right]$ & $K_{y y}\left[\mathrm{~mm}^{2}\right]$ & $K_{z z}\left[\mathrm{~mm}^{2}\right]$ \\
\hline 0.9 & 4.3 & 1.7 & 0.0269 & 0.00145 & 0.00426
\end{tabular}

The PIV measurements are carried out at the symmetry plane of the test section. The measured bulk Reynolds numbers $\operatorname{Re}_{b}=U_{b} H / \mathrm{v}$, where $U_{b}$ is the bulk mean velocity and $\mathrm{v}$ is the kinematic viscosity of the fluid, are 3000,5000,7500,10000 and 15000 . 


\section{Results and discussions}

\subsection{Streamwise velocities}

As shown in figure 2, the distribution profiles of the streamwise mean velocities look almost symmetrical. This indicates that the effects of the porous medium are not significant and almost the same as those of the smooth wall. Note that $y / H=0$ and 1.0 correspond to the porous-bottom and solid- smooth-top surface locations, respectively.

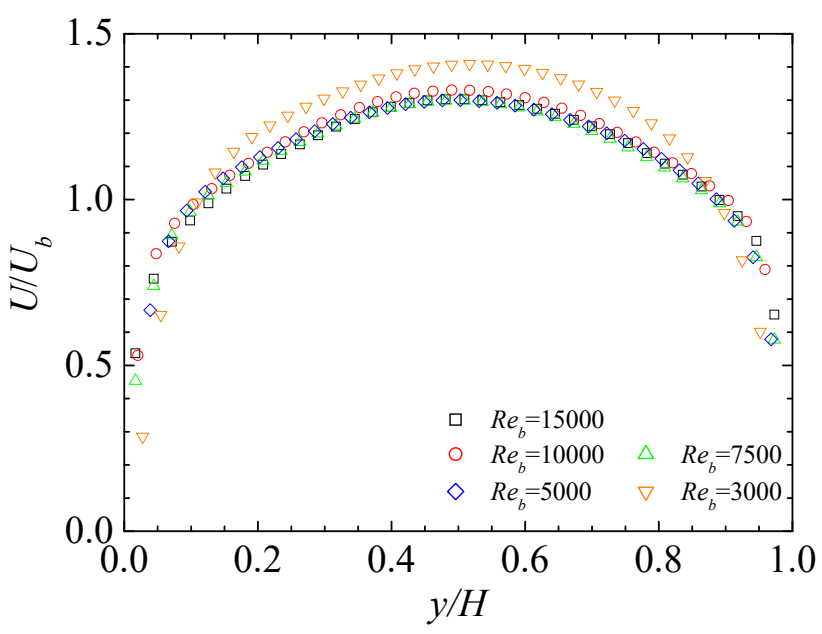

Figure 2: Streamwise mean velocity distributions.

To investigate the difference between the porous and the solid wall sides in more detail, profiles are compared in the semi-logarithmic chart. Since the present study does not measure the wall friction, the streamwise friction velocity $u_{\tau}$ is calculated by the correlation proposed by Gavrilakis (2019) referring to the DNS results for rectangular ducts. For the fully developed straight square duct, the Darcy-Weisbach friction factor $f$ may be written as

$$
f=\frac{8 u_{\tau}^{2}}{U_{m}^{2}}
$$

Note that $u_{\tau}$ represents the averaged friction velocity. The simulation data of fully developed duct flows by Gavrilakis (2019) collapse to the power law

$$
f=0.281 R e_{m}^{-1 / 4.13} .
$$

Using eqs.(1) and (2), $u_{\tau}$ is calculated to normalize the present data. Figure 3 shows the mean velocity $U^{+}\left(=\frac{U}{u_{\tau}}\right)$ profiles normalized by $u_{\tau}$ as a function of the normalized wall-normal distance $y^{+}=\frac{y u_{\tau}}{v}$. The dashed lines and the two-dot chain lines express the DNS data by Pirozzoli et al. (2018) for reference purposes and the solid lines in figure 3 express the logarithmic law of the velocity

$$
U^{+}=\frac{1}{\kappa} \ln y^{+}+5.5
$$

where $\kappa$ is the von Kármán constant of 0.41 . Figure 3 indicates that the present data at $R e_{b}=7500$ are well accord with the DNS data at $R e_{b}=7000$. This implies that the estimated friction by eq.(2) reasonably represents the present measurements. As can be seen in figure 3 , the $U^{+}$distributions of the porous and the solid wall sides are almost identical at $R e_{b}=3000-7500$, while that of the porous wall side is obviously below that of the solid wall side at $R e_{b}=15000$. It is also recognized that the profile of the porous wall side seems slightly below that of the solid wall side at $R e_{b}=10000$. Note that the downward shift of the mean velocity (in the semi-logarithmic chart) corresponds to the increase of the wall friction. From this trend, it 


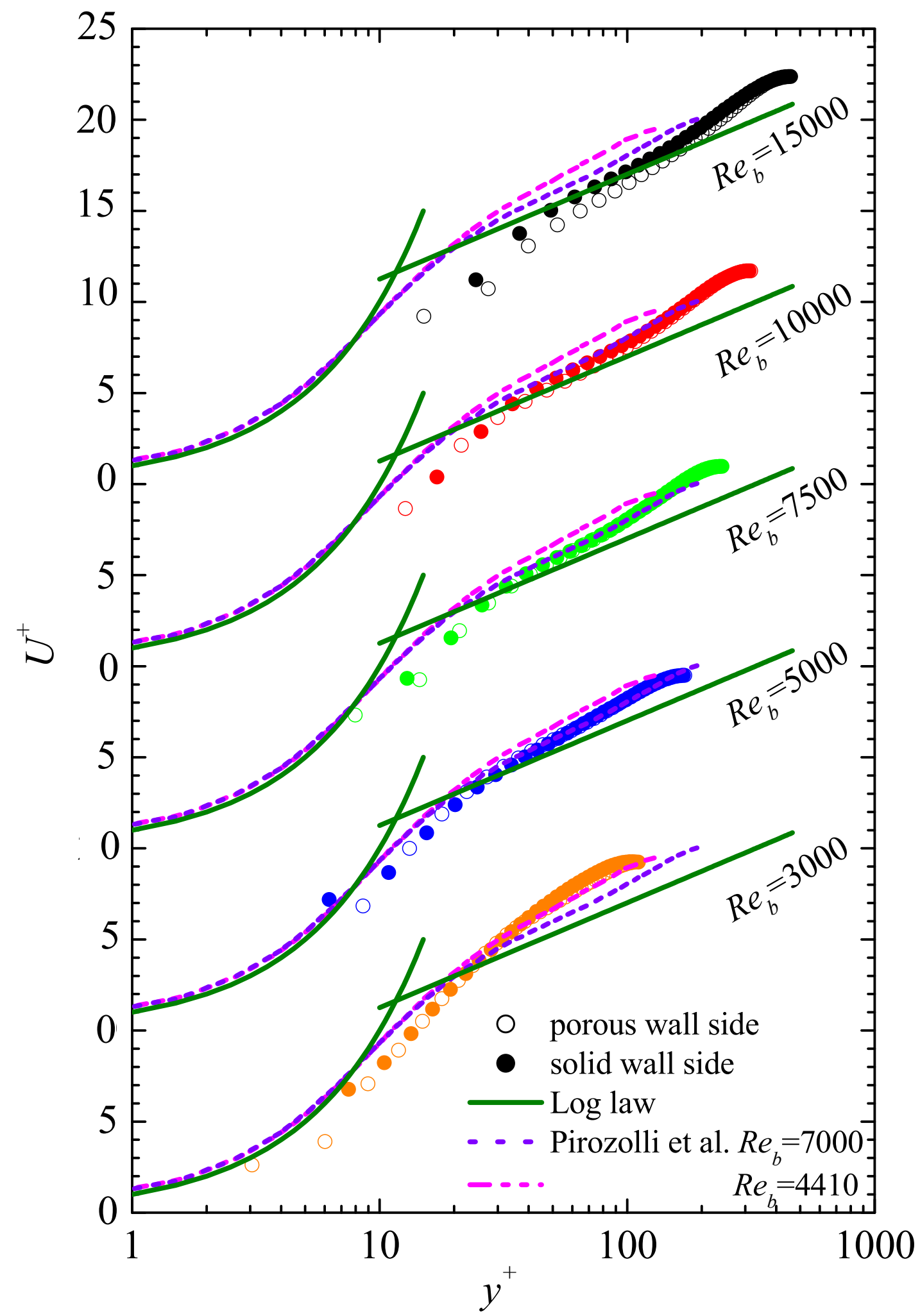

Figure 3: Comparison of the streamwise mean velocities. 
can be expected that when $R e_{b} \lesssim 10000$, the porous wall friction is comparable to that of the smooth surface, and it gradually increases with the Reynolds number at $R e_{b}>10000$.

Gómez-de Segura and García-Mayoral (2019) argued whether the porous media reduced the turbulent drag depending on the wall-normal permeability Reynolds number $\sqrt{K_{y y}^{+}}=\frac{u_{\tau} \sqrt{K_{y y}}}{v}$. They simulated that the maximum drag reduction occurred at $\sqrt{K_{y y}^{+}} \approx 0.38$ for $\psi_{x y}=3.6$ and 5.5 while the drag reduction effect became extinct at $\sqrt{K_{y y}^{+}}>0.6$. The values of $\sqrt{K_{y y}^{+}}$at $R e_{b} \leq 10000$ in the present study are in the range of the drag reduction suggested by Gómez-de Segura and García-Mayoral (2019) as shown in table 2 while at $R e_{b}=15000$ the value of $\sqrt{K_{y y}^{+}}$does not satisfy the condition of the drag reduction. When $\sqrt{K_{y y}^{+}}$satisfies the drag reduction condition, the effect of the present streamwise preferential porous medium can be expected to be similar to that of a smooth wall, and it does not drastically reduce the turbulent skin friction as Gómez-de Segura and García-Mayoral (2019) predicted. In case that $\sqrt{K_{y y}^{+}}$does not satisfy the condition, the friction increases as predicted.

Table 2: Permeability Reynolds numbers.

\begin{tabular}{c|ccccc}
$R e_{b}$ & 3000 & 5000 & 7500 & 10000 & 15000 \\
\hline$\sqrt{K_{y y}^{+}}$ & 0.16 & 0.26 & 0.36 & 0.48 & 0.69
\end{tabular}

\subsection{Turbulence quantities}

Figure 4 presents the root mean square (r.m.s.) velocities and the Reynolds shear stress distributions. The profile at each Reynolds number looks quite symmetry. This tendency is similar to that of the aforementioned streamwise velocities.

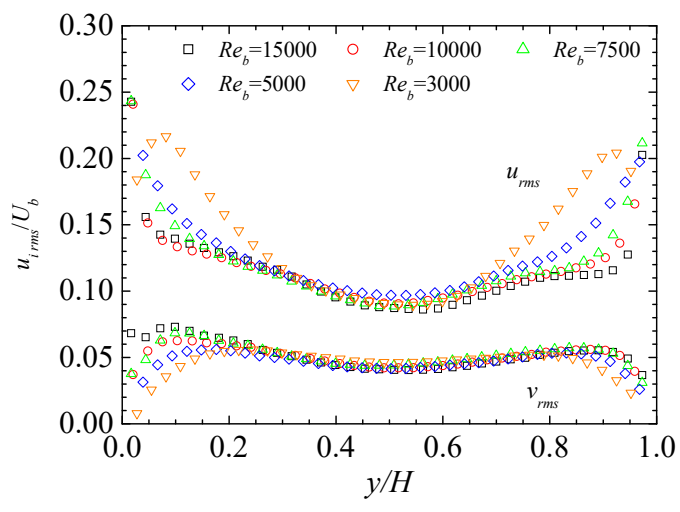

(a) r.m.s velocities

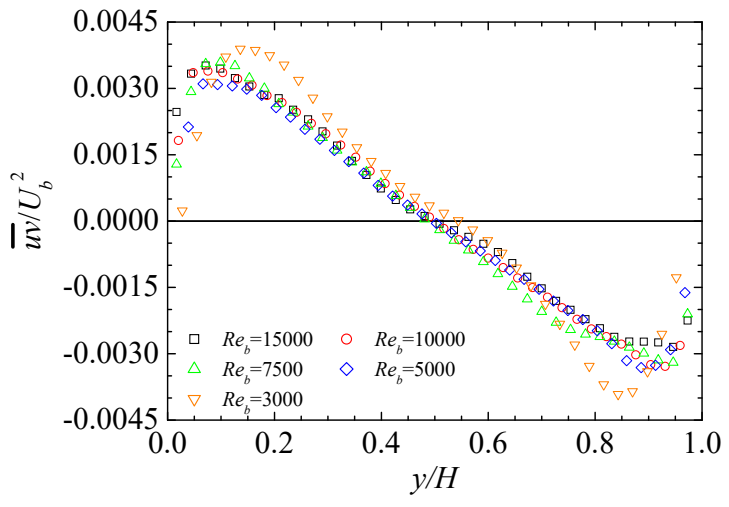

(b) Reynolds shear stresses

Figure 4: Turbulence quantity distributions.

To see the difference of the r.m.s. velocities between the porous and solid wall sides, figure 5 compares the r.m.s. velocities: $u_{r m s}$ and $v_{r m s}$, at $R e_{b}=5000$ and 15000. At $R e_{b}=5000$, as shown in figure 5(a), the observed tendency is the same as that suggested by the velocity distribution, i.e., there is no increase of turbulence on the porous wall side. At $R e_{b}=15000$, as shown in figure 5(b), r.m.s. velocities of the porous side are larger than that of the solid side and this trend is also the same as that of the velocity distribution. 


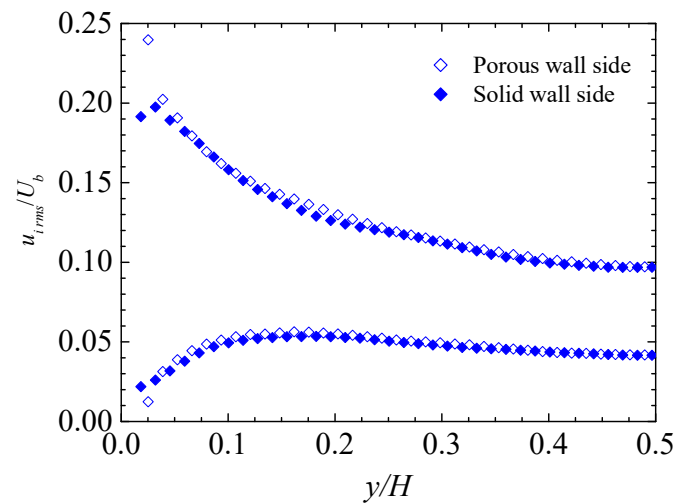

(a) $R e_{b}=5000$

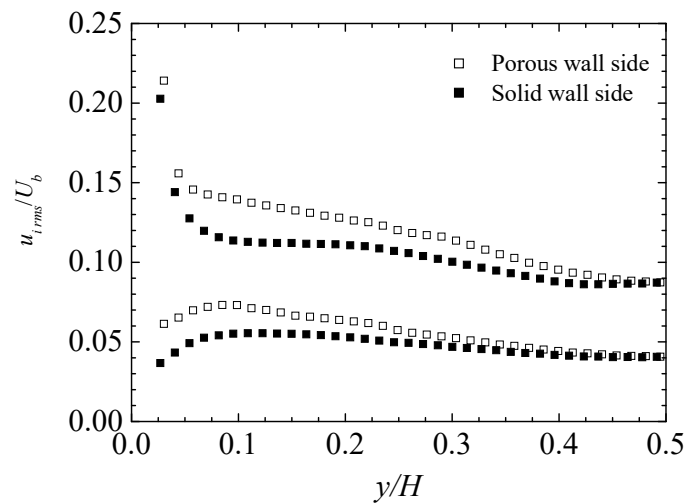

(b) $R e_{b}=15000$

Figure 5: Comparison of the r.m.s. velocities between the porous and solid wall sides.

As for the Reynolds shear stresses shown in figure 6, at $R e_{b}=5000$ both profiles of porous and solid wall sides are at the same level while at $R e_{b}=15000$ the Reynolds shear stress of the porous wall side is higher than that of the solid wall side. These trends are consistent with those observed in the r.m.s. velocities. The trend at $R e_{b}=15000$ is consistent with the fact that the semi-logarithmic velocity over the porous wall profile is lower than that on the solid wall side. We assume that this difference may come from the development of the secondary flows which we will investigate in due course.

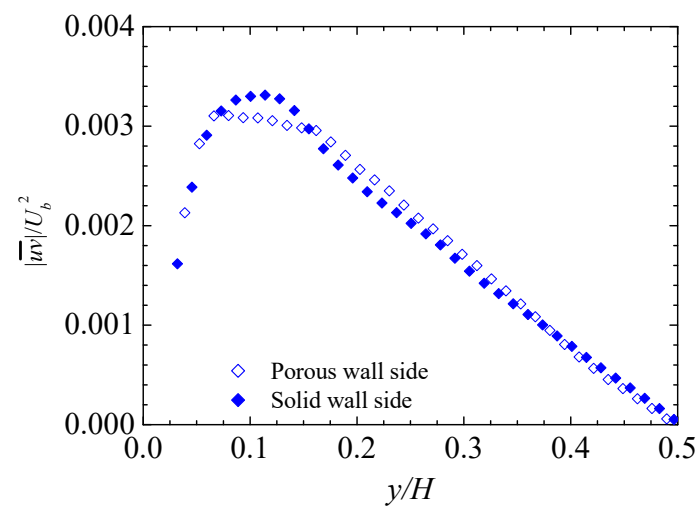

(a) $R e_{b}=5000$

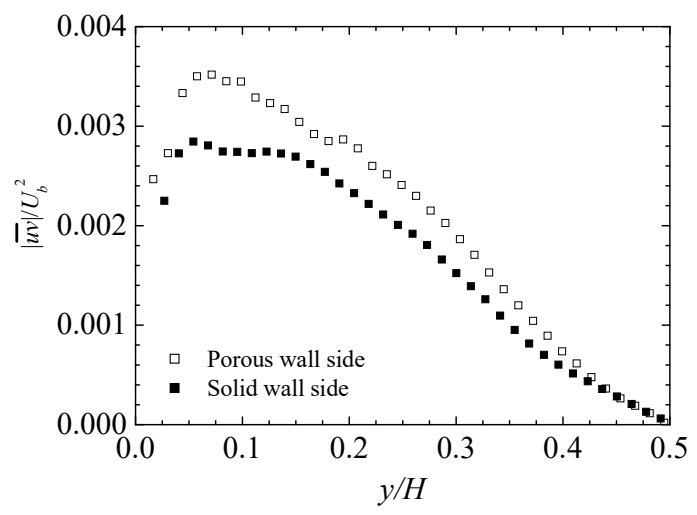

(b) $R e_{b}=15000$

Figure 6: Comparison of the Reynolds shear stresses between the porous and solid wall sides.

In order to discuss bursts which contribute to the formation of turbulent flow energy and the Reynolds stress, Fig.7 compares the decomposed Reynolds shear stress profiles at $R e_{b}=5000$ and 15000 using the quadrant analysis method of Willmarth and Lu (1972):

$$
Q_{m}=\frac{1}{N} \sum\left(u^{\prime} v^{\prime}\right)_{m}
$$

where subscript $m(=1-4)$ corresponds to the quadrant event. It is seen that the contributions of the second quadrant $\left(Q_{2}\right)$ : ejections, are significantly larger on the porous wall side than those on the solid wall side, both at $R e_{b}=5000$ and $R e_{b}=15000$. It is thus considered that the vortex structure near the porous wall is different from that near the solid wall. Furthermore, it is interesting that the third quadrant $\left(Q_{3}\right)$ : inward interactions, clearly dominates over the first quadrant $\left(Q_{1}\right)$ : outward interactions, on the porous wall side at 
$R e_{b}=15000$. We assume that these trends may relate to the secondary flows, and hence we plan to perform PIV measurements at different spanwise sections to confirm this.

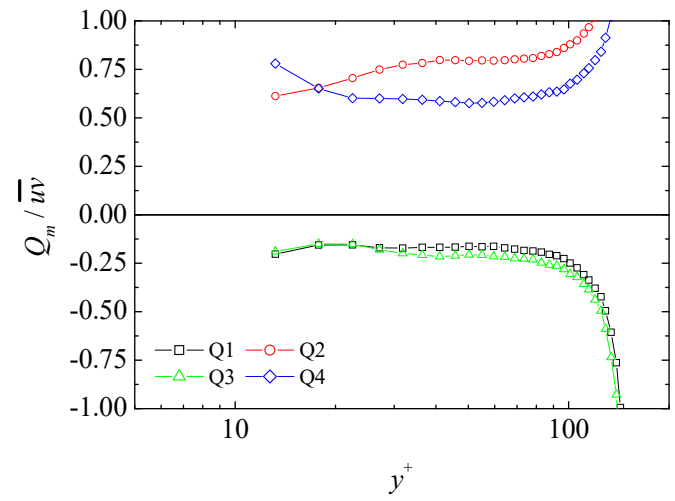

(a) Porous wall side at $\operatorname{Re}_{b}=5000$

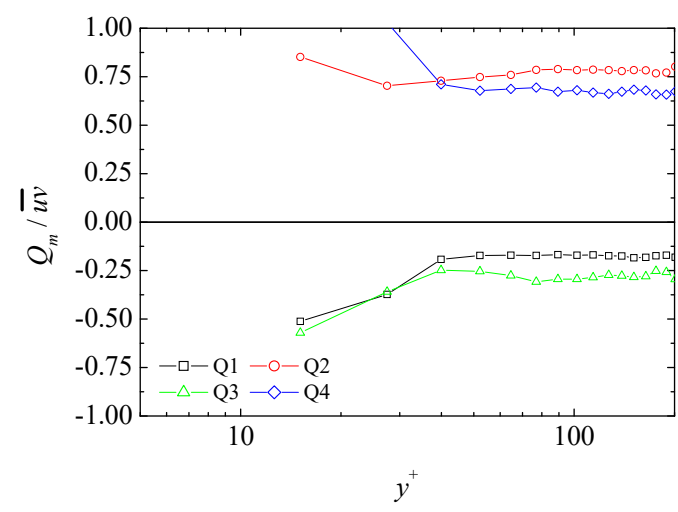

(c) Porous wall side at $R e_{b}=15000$

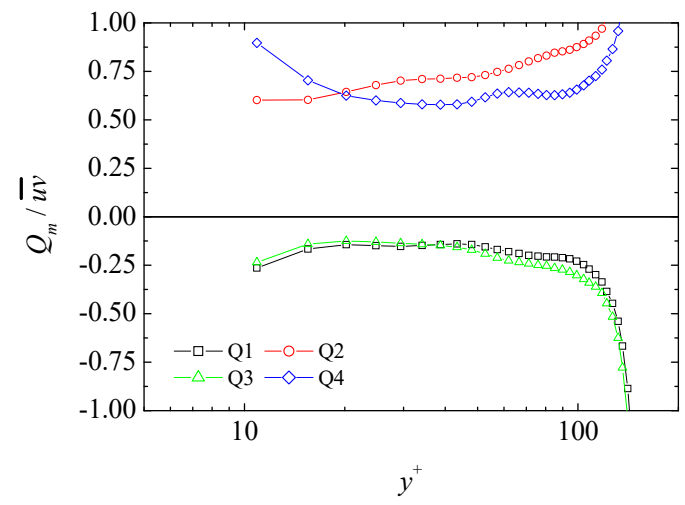

(b) Solid wall side at $R e_{b}=5000$

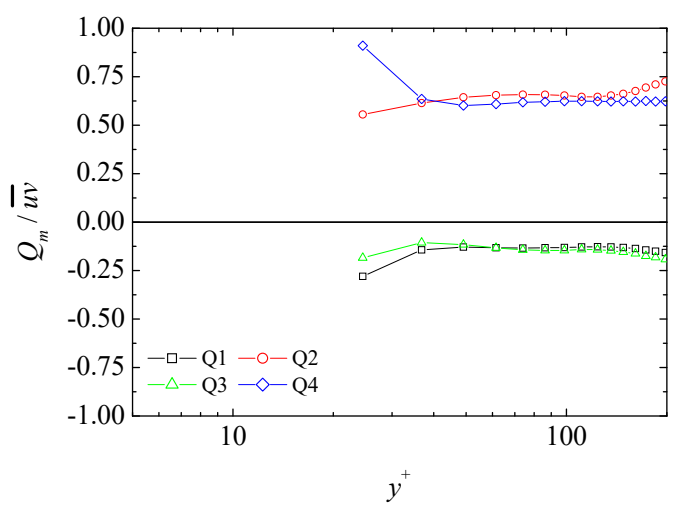

(d) Solid wall side at $R e_{b}=15000$

Figure 7: Quadrant analyses of the Reynolds shear stress distribution at $R e_{b}=5000$ and $R e_{b}=15000$.

The reason why the present porous wall behaves like a smooth wall at $R e_{b}<10000$ is assumed that the normalized mesh size $G^{+}=\frac{u_{\tau} G}{v}$ and the normalized wire diameter $d^{+}=\frac{u_{\tau} d}{v}$ are sufficiently small enough to be negligible. (It is known that when the roughness height is of the same order or smaller than 5, the wall roughness hardly affects turbulence.) As shown in table 3, $G^{+}$and $d^{+}$are small enough to be ignored. At $R e_{b}=15000$, it is considered that the flow through porous medium affects turbulence generation even though $G^{+}$and $d^{+}$are smaller than 5 .

Table 3: Normalized mesh size and wire diameter.

\begin{tabular}{c|ccccc}
$R e_{b}$ & 3000 & 5000 & 7500 & 10000 & 15000 \\
\hline$G^{+}$ & 0.653 & 1.05 & 1.47 & 1.93 & 2.79 \\
$d^{+}$ & 0.424 & 0.679 & 0.957 & 1.25 & 1.81
\end{tabular}




\section{Concluding remarks}

To understand the turbulence characteristics of a streamwise-preferential porous medium, PIV measurements have been carried out. Fully developed turbulent square duct flows over a porous medium whose permeability ratio of the wall-normal to streamwise permeabilities is $\psi_{x y}=4.3$ are discussed at $\operatorname{Re}_{b}=$ $3000,5000,7500,10000$ and 15000 . The presently obtained major remarks are:

I. At $\operatorname{Re}_{b}<10000$, the mean velocity profiles suggest that the wall friction on the present porous wall is nearly the same as the smooth wall friction while at $R e_{b}>10000$ the porous wall friction tends to be larger than the smooth wall friction.

II. At $R e_{b}=5000$, the r.m.s. velocity and the Reynolds stress distributions of the porous and solid wall sides are almost the same, while at $R e_{b}=15000$, both distributions of the porous side are larger than those of the solid wall side. The turbulence trend at $R_{b}=15000$ indicates the increase of the wall wall friction on the porous side, which is consistent with the trend of the semi-logarithmic velocity distributions.

III. In the range of all measured Reynolds numbers, even at $R e_{b}=15000$ where the friction increases on the porous side, the normalized mesh size and the normalized wire diameter are considered to be small enough to be ignored. This trend suggests that the turbulence is affected by the flow through the porous medium.

Although Gómez-de Segura and García-Mayoral (2019) showed drastic drag reduction by streamwise preferential porous media in their simulation, it is not observed in the present experiments. It was found that in their drag reducing condition, the present streamwise-preferential porous medium produces the friction as much as the smooth surface does.

\section{Acknowledgements}

A part of this study was financially supported by the research grant (No. 19H02069) of the JSPS. The authors thank Dr. Kaneda for supporting the experiments.

\section{References}

Breugem WP, Boersma BJ, and Uittenbogaard RE (2006) The influence of wall permeability on turbulent channel flow". J Fluid Mech 562:35-72

Gavrilakis S (2019) Post-transitional periodic flow in a straight square duct. J Fluid Mech 859:731-753

Gómez-de Segura G and García-Mayoral R (2019) Turbulent drag reduction by anisotropic permeable substrates - analysis and direct numerical simulations. J Fluid Mech 875:124-172

Lovera F and Kennedy JF (1969) Friction factors for flat bed flows in sand channels. J Hydr Div, ASCE 95:1227-1234

Manes C, Poggi D, and Ridol L (2011) Turbulent boundary layers over permeable walls: scaling and nearwall structure. J Fluid Mech 687:141-170

Manes C, Pokrajac D, McEwan I, and Nikora V (2009) Turbulence structure of open channel flows over permeable and impermeable beds: A comparative study. Phys Fluids 21:125109

Pirozzoli S, Modesti D, Orlandi P, and Grasso F (2018) Turbulence and secondary motions in square duct flow. J Fluid Mech 840:631-655

Pokrajac D and Manes C (2009) Velocity measurements of a free-surface turbulent flow penetrating a porous medium composed of uniform-size spheres. Transp Porous Media 78:367-383

Rosti ME, Brandt L, and Pinelli A (2018) Turbulent channel flow over an anisotropic porous wall-drag increase and reduction. J Fluid Mech 842:381-394 
Suga K (2016) Understanding and modelling turbulence over and inside porous media. Flow Turb Combust 96:717-756

Suga K, Matsumura Y, Ashitaka Y, Tominaga S, and Kaneda M (2010) Effects of wall permeability on turbulence". Int J Heat Fluid Flow 31:974-984

Suga K, Mori M, and Kaneda M (2011) Vortex structure of turbulence over permeable walls. Int J Heat Fluid Flow 32:586-595

Suga K, Nakagawa Y, and Kaneda M (2017) Spanwise turbulence structure over permeable walls. J Fluid Mech 822:186-201

Suga K, Okazaki Y, and Kuwata Y (2020) Characteristics of turbulent square duct flows over porous media. J Fluid Mech 884. a7

Willmarth WW and Lu SS (1972) Structure of the reynolds stress near the wall. J Fluid Mech 55:65-92

Zippe HJ and Graf WH (1983) Turbulent boundary-layer flow over permeable and non-permeable rough surfaces. J Hydraul Res 21:51-65 